\title{
Assessment of Stationarity Horizon of the Heart Rate
}

\author{
Gilles Thonet ${ }^{1}$, Thierry Duvanel ${ }^{1}$, Jean-Marc Vesin ${ }^{1}$, Etienne Pruvot ${ }^{2}$, Martin Fromer ${ }^{2}$, \\ and the HRVF-study investigators
${ }^{1}$ Signal Processing Laboratory, Swiss Federal Institute of Technology, 1015 Lausanne, Switzerland ${ }^{2}$ Division of Cardiology, University Hospital, 1011 Lausanne, Switzerland e-mail: thonet@ltssg4.epfl.ch

\begin{abstract}
This work presents a new method using time-varying autoregressive modelling for the assessment of heart rate signals stationarity in patients before the onset of ventricular tachyarrhythmias, including comparison with a control group. A general stationarity trend is reported for all subjects, and particularly no significant change is observed before an arrhythmic event. Evaluation of the model fitting performed by a hypothesis test suggests the presence of nonlinearities.
\end{abstract}

\section{INTRODUCTION}

Heart rate (HR) variability analysis is a well known technique to study the interaction between the autonomic nervous system and the heart sinus pacemakers. However, classical linear methods (DFT, AR modelling) relie on the assumption of stationarity. This hypothesis is not obvious since long-term HR recordings have shown strong circadian variations, suggesting a nonstationary behaviour. Numerous studies attempted to find out some particular features of the HR dynamics preceding the onset of ventricular tachyarrhythmias (VTA). However, results are still contradictory and require further investigations.

The purpose of this work is to present a new method for evaluating the stationarity of the HR signal and therefore to highlight possible changes in its structure before an arrhythmic event. Moreover, results are compared to control subjects in upright position.

The proposed method is directly derived from a parametric approach. We consider a time-varying autoregressive (TVAR) model with a decomposition of the AR coefficients variation on a set of basis functions. Stationarity is assessed by the determination of optimal orders using Rissanen's MDL criterion. Then, statistical significance of the TVAR coefficients is evaluated by hypothesis testing.

\section{Time-Varying Autoregressive Model}

A discrete-time stochastic process $x(n)$ is often expressed by an autoregressive (AR) model with $p$ coefficients $a_{i}$. When $x(n)$ is not stationary, this approach is no longer valid, since there is no time dependence in the coefficients $a_{i}$. A solution proposed by Rao [1] is to make the assumption that the variations of $a_{i}$ can be approximated by a linear combination of a finite number of known deterministic functions $u_{k}$ (called basis): $a_{i}(n)=\sum_{k=0}^{q} a_{i k} u_{k}(n)$.
Therefore, the TVAR model has the following form:

$$
x(n)=\sum_{i=1}^{p}\left(\sum_{k=0}^{q} a_{i k} u_{k}(n)\right) x(n-i)+\epsilon(n)
$$

and estimation of the coefficients $a_{i k}$ is performed by minimization of the mean square error. Grenier [2] and Hall \& al. [3] have described with more details the identification of this model and its computational aspects.

\section{Parameters Selection and Model Va- LIDATION}

The TVAR model makes use of three degrees of freedom: the choice of the basis functions $u_{k}$, the AR order $p$ and the basis order $q$.

Many different solutions have been proposed in the literature for the functions $u_{k}([2],[3])$. None of them seems to be definitive, partly because the choice of $u_{k}$ needs some a priori knowledge upon the signal time variations. We consider here classical polynomial functions (namely Chebycheff, Hermite and Legendre).

For determination of $p$ and $q$, we make use of Rissanen's Minimum Description Length (MDL) criterion [4], which can be generalized in our case:

$$
\operatorname{MDL}(p, q)=N \ln \left(\hat{\sigma}_{p, q}^{2}\right)+p(q+1) \ln (N)
$$

where $N$ is the length of the signal, and $\hat{\sigma}_{p, q}^{2}$ is the residual error variance.

Then, we achieve model validation using a hypothesis test on each coefficient $a_{i k}$. For that, we need to estimate the distribution of the TVAR coefficients, which can be performed by deriving the expression of the maximum likelihood estimator $L(a)$. Under certain mild conditions, this estimator is known to be asymptotically normally distributed [5]. The diagonal elements of the inverse of $\mathbf{F}=\partial^{2} \log \mathbf{L}(\mathbf{a}) / \partial \mathbf{a} \partial \mathbf{a}^{\mathbf{T}}$ are the coefficients variances $\sigma_{a_{i k}}^{2}$.

Therefore, under the hypothesis that a non-significant coefficient is zero (null hypothesis), we can compute the probability $\operatorname{prob}\left(\left|a_{i k}\right| \leq d \sigma_{a_{i k}}\right)=\operatorname{prob}\left(\left|a_{i k} / \sigma_{a_{i k}}\right| \leq d\right)$.

According to the value of $d$, we accept the hypothesis with a significance level $\alpha$ given by the normal distribution $N(\mu=0, \sigma=1)$. We selected a standard value $\alpha=0.95$, leading to a minimum ratio $\left|a_{i k} / \sigma_{a_{i k}}\right|$ of 1.96 . 


\section{Stationarity Assessment}

In order to evaluate the stationarity of the signal, the TVAR model is first applied for different values of $p$ and $q$. The MDL criterion is then computed for each couple $(p ; q)$ and minimized to obtain the optimal orders. Finally, the number of statistically significant coefficients is derived.

If the basis order $q$ selected by the MDL criterion is high (typically greater than 2 or 3 ), it means that a large number of basis functions is required to accurately model the signal. It implies also that statistical properties of the signal are strongly changing with time, suggesting a highly nonstationary behaviour. On the other hand, if $q$ equals zero, we can expect that the signal structure is closer to stationarity, since a TVAR model in this case is equivalent to a classical AR model. The statistical significance of the coefficients provides in this context a measure of the model fitting to the signal.

Moreover, it is often useful to determine if a smaller part of the signal can be considered stationary. For this purpose, we apply also the described method to increasing sections of the signal in order to evaluate the horizon of stationarity.

\section{Application to HR Signals}

The proposed method has been applied to HR recordings coming from two different populations. The first one includes 7 patients (mean age: $54 \pm 14$, mean ejection fraction: $18.2 \pm 11.4 \%$ ), suffering from VTA. We retrieved from Medtronic $7218 / 7220$ defibrillators two sets of 7 HR series consisting of $1024 \mathrm{RR}$ intervals with less than $5 \%$ of ventricular premature contractions. These data were obtained from a multicentric investigation including 8 European centers (HRVF-study). The first set is composed of RR samples preceding the onset of a VTA (mean cycle length: $334 \pm 40 \mathrm{~ms}$ ), while the second one concerns RR samples acquired under baseline condition. On the other hand, 3 normal subjects constitute the control group, where data have been gathered during an orthostatic stress.

The following parameters have been used for simulations: Legendre basis, TVAR order $p$ covering the range 10 to 20 and basis order $q$ ranging from 0 to 5 . Each signal is divided into 6 sections of increasing duration (with a step size of $100 \mathrm{~s}$ ), in order to examine the horizon of stationarity. An example is presented for a patient belonging to the first population (before VTA and in baseline condition) and for a control subject. The following table provides for 3 durations ( $200 \mathrm{~s}, 400 \mathrm{~s}$ and $600 \mathrm{~s}$ ) the optimal orders $(p ; q)$ obtained with the MDL criterion and the corresponding significance rate $(\mathrm{SR}=$ number of significant coefficients/total number of TVAR coefficients).

\begin{tabular}{||c||c|c||c|c||c|c||}
\hline \hline Population & p;q & SR & p;q & SR & p;q & SR \\
\hline \hline VTA & $10 ; 0$ & 0.30 & $10 ; 0$ & 0.40 & $10 ; 0$ & 0.60 \\
\hline Baseline & $10 ; 0$ & 0.50 & $10 ; 0$ & 0.40 & $10 ; 0$ & 0.40 \\
\hline Control & $10 ; 0$ & 0.30 & $10 ; 0$ & 0.30 & $10 ; 0$ & 0.30 \\
\hline \hline
\end{tabular}

We have observed that the optimal basis order $q$ is nearly always equals to zero for all groups and conditions. The strong time constancy of the optimal orders suggests that up to a 10-minute duration, the signal structure is stable and accordingly very close to stationarity. Therefore, results invalidate the assumption of a modification of the HR dynamics preceding onset of VTA. Then, we have compared the number of significant coefficients between recordings in baseline condition and recordings preceding VTA in patients suffering from arrhythmic events. The application of a non-parametric test (Wilcoxon signed-rank) suggests that the significance rate is higher before a VTA than under baseline condition $(p=0.07)$. Therefore, the HR dynamics preceding VTA seems to be more linear, but this should be validated on a larger population. In a second step, we have noticed again that the significance rate appears to be lower for control subjects than for patients before VTA (Mann-Whitney U: $\mathrm{p}=0.06$ ). Consequently, our study reveals an increasing trend in the linearity of HR signals, from normal subjects to patients before an arrhythmic event.

Accordingly, results show that a TVAR model does not fit HR signals better than a classical AR model. However, if we note that few coefficients are statistically significant, it is clear that this kind of signal cannot be accurately described by classical linear models. Consequently, nonlinear modelling of the HR signal should be investigated.

\section{CONCLUSION}

In this work, we have developed a new method using TVAR modelling for the evaluation of HR signals stationarity. Optimal orders for this model have been selected by Rissanen's MDL criterion and hypothesis testing has been introduced to assess the statistical significance of the TVAR coefficients.

In our two groups (control subjects and arrhythmic patients), a stationarity trend has been highlighted. In particular, no modification of the HR dynamics preceding an arrhythmic event has been observed. The comparison of the TVAR coefficients significance rate has suggested an increasing linearity of HR signals from control subjects to patients suffering from a ventricular tachyarrhythmia. Nevertheless, the weak average level of coefficients significance indicates a probable nonlinear component and justifies further studies using nonlinear tools.

\section{REFERENCES}

[1] T. S. Rao. The fitting of nonstationary time-series models with time-dependent parameters. J. Royal Statist. Soc. Series $B, 32(2): 312-322,1970$.

[2] Y. Grenier. Time-dependent arma modeling of nonstationary signals. IEEE Trans. Acoust., Speech, and Signal Processing, 31(4):899-911, 1983.

[3] M. G. Hall, A. V. Oppenheim, and A. S. Willsky. Timevarying parametric modeling of speech. Signal Processing, $5: 267-285,1983$.

[4] J. Rissanen. Stochastic Complexity in Statistical Inquiry. World Scientific, 1989.

[5] N. L. Johnson and F. C. Leone. Statistics and Experimental Design, volume 1. John Wiley and Sons, 2nd edition, 1976. 